\title{
CT Measurement of Proximal Femoral Medullary Cavity Morphology in Healthy Subjects from Northern China
}

\section{Ran Zhao}

Peking University Third Hospital https://orcid.org/0000-0003-3534-5570

Hong Cai ( $\square$ caihong_76@126.com )

Peking University Third Hospital

\section{Ke Zhang}

Peking University Third Hospital

Hua Tian

Peking University Third Hospital

\section{Research article}

Keywords: proximal femoral medullary, CT measurement, morphology

Posted Date: September 10th, 2020

DOI: https://doi.org/10.21203/rs.3.rs-55403/v1

License: (c) (i) This work is licensed under a Creative Commons Attribution 4.0 International License. Read Full License 


\section{Abstract}

Purpose: In total hip arthroplasty (THA), optimal stability and fixation is achieved via tight adaptation of the prosthesis to the bone. This study aimed to analyze the proximal femoral medullary cavity morphology of Chinese subjects on three-dimensional reconstructed imagery to explore the factors influencing these parameters.

Methods: CT was performed on 63 healthy subjects from northern China (94 hips; 31 men, 63 women). Three-dimensional CT reconstruction of the proximal femoral medullary cavity was performed using Mimics 22.0 software. The anatomical parameters related to THA were measured to examine the relationships between sex, age, and femoral length. These parameters were compared with previously published data.

Results: The internal and external coronal diameters of the femoral medullary cavity, and the anterior and posterior sagittal diameters had different contracting trends. There were correlations between the femoral length, femoral head diameter, and coronal diameter, and between the sagittal diameter and crosssectional area of the medullary cavity. Age was negatively correlated with the CFI(canal flare index). Sex was correlated with the cross-sectional coronal/sagittal diameters, but not with the canal flare index. The proximal end of the medullary cavity was similar to that reported in Caucasians, but the Asian medullary cavity was narrower distal to the level of the lesser trochanteric line.

Conclusions: THA-related anatomy was affected by sex, age, and subpopulation. Compared with sex, age had a greater effect on the fit of the stem to the femoral canal. Each patient must be treated individually, as the anatomical structure varied between individuals.

\section{Introduction}

Total hip arthroplasty (THA) effectively treats a variety of hip diseases, with good long-term effects [1]. The success of cementless THA depends on a close geometric fit between the prosthesis and bone in order to achieve optimal primary stability and secondary biologic fixation [2]. Modern cementless prothesis stems engage the medial and lateral cortices of the femoral medullary metaphysis through a tapered wedge, and match the metaphyseal medullary cavity morphology as much as possible [3]. Insufficient matching may lead to loosening of the prosthesis due to a lack of proximal osseointegration [4].

Currently, most THA prostheses are designed based on the parameters of the medullary cavity of the Caucasian population. However, the shape of the medullary cavity is significantly influenced by ethnicity [5]. Most studies evaluating the morphology of the proximal femoral medullary cavity in Asians are based on two-dimensional studies [6, 7], with less studies based on three-dimensional (3D) CT [8]. Furthermore, there is a lack of 3D studies on the morphology of the medullary cavity of subjects from northern China. The purpose of the present study was to use 3D CT imagery to: (1) describe the anatomical morphology of the proximal femoral medullary cavity and the parameter variation interval of the northern Chinese 
population; (2) determine the correlations between proximal femoral medullary cavity parameters and demographic data, such as sex, age, and femoral length; (3) compare the present data with previously published data.

\section{Materials And Methods \\ 2.1 Study population}

This study was approved by the Ethics Committee of our institution, and each study participant provided written informed consent. The selected population was a non-randomized group of healthy volunteers. The inclusion criteria were healthy adults with no history of lower limb disease. Exclusion criteria were: hip symptoms such as pain, deformity, abnormal movement, or claudication; hip diseases such as fracture, osteoarthritis, aseptic avascular necrosis of the femoral head, developmental dysplasia of the hip, or history of lower limb surgery; pregnancy or lactation.

A total of 63 adults (94 hips) were recruited, comprising 21 men (31 hips) and 42 women (63 hips). The average age was 51.5 years (range 23 to 68 years). All participants were born in northern China. The study procedures were performed in accordance with the Helsinki Declaration of 1975, revised in 2000.

\subsection{CT parameters}

All participants underwent 64-slice CT scanning (Discovery 750 HD; GE Healthcare, Madison, WI, USA) using the same projection protocol. The parameters were: $120 \mathrm{kV} ; 80 \mathrm{mAs}$; reconstruction thickness, $1.0 \mathrm{~mm}$; interlayer spacing, $1.0 \mathrm{~mm}$. Each participant was placed in supine position with the lower limbs fixed in a neutral position, fully extended and slightly rotated, with the toes pointing upward. The lower limbs were fixed, and seat belts were fastened to prevent movement during the CT process. Participants were scanned from the iliac crest to the knee joint. The images were saved as DICOM images.

\subsection{Three-dimensional image reconstruction and measurements}

DICOM data from CT scans were imported into Mimics 22.0 software (Materialise, Leuven, Belgium) for $3 \mathrm{D}$ reconstruction. The skeletal structure was calculated automatically in accordance with the radiodensity. The contours of sequential images were connected by triangular facets to establish a 3D model that could then be displayed in any position or direction. The software was used to perform the following steps to create the femoral medullary cavity morphology: contrast adjustment (0-350), thresholding $(226-1,821)$, region growing, mask editing, and mask editing in 3D to obtain the femoral cortical bone. Three-dimensional reconstruction of the femur and medullary cavity was performed with the CT bone module, and the proximal femoral medullary cavity morphology was obtained with Boolean operations, cavity filling, morphology operations, and 3D calculations. The center of rotation of the femoral head was derived by the fitting of a sphere on the surface of the femoral head. The fit centerline was calculated from the femoral medullary cavity rather than the femoral cortex. 
The reconstruction of the coordinate system is shown in Fig. 1. The line connecting the center point of the medullary cavity at $20 \mathrm{~mm}$ proximal to the lesser trochanteric line (T-20) and $60 \mathrm{~mm}$ distal to the lesser trochanteric line $(T+60)$ was used as the approximate central axis of the proximal femoral medullary cavity. The plane perpendicular to the central axis of the proximal femur was defined as the horizontal plane; the plane consisting of the center point of the fitting sphere of the femoral head and the central axis of the proximal femoral medullary cavity was defined as the frontal plane; the plane perpendicular to the frontal plane and the horizontal plane was defined as the sagittal plane.

One of the classical methods used to measure the morphology of the medullary cavity of the proximal femur is the noble measurement method [9]. The noble measurement method describes the medullary cavity morphology by measuring the cortical width in the frontal plane of the medullary cavity and calculating the ratio of the proximal medullary cavity to the isthmus, which is known as the canal flare index (CFI). As most modern prosthetic stems are short and cannot reach the level of the isthmus, many improved definitions of the CFI have been proposed. Boymans et al. proposed the concept of the CFI60 as the medullary diameter at the level of $20 \mathrm{~mm}$ proximal to the lesser trochanteric line divided by that at the level of $60 \mathrm{~mm}$ distal to the lesser trochanteric line [10].

The parameters measured in the present study are described below.

(1) Measurement of parameters in the proximal horizontal plane of the femoral medullary cavity (Fig. 2).

The level of the lesser trochanteric line was set as layer $0(T)$. The cross-sectional parameters were then measured every $5 \mathrm{~mm}$ from the level of $25 \mathrm{~mm}$ proximal to the lesser trochanteric line (T-25) to the level of $20 \mathrm{~mm}$ distal to the lesser trochanteric line $(T+20)$, and every $10 \mathrm{~mm}$ from $T+20$ to the level $60 \mathrm{~mm}$ distal to the lesser trochanteric line $(T+60)$.

The horizontal medullary cavity measurement parameters are shown in Fig. 3. The coronal medial diameter (M) was defined as the distance from the central axis to the medial cortex of the coronal plane, while the coronal lateral diameter $(L)$ was defined as the distance from the central axis to the lateral cortex of the coronal plane. The coronal medial-lateral diameter $(M L)$ was defined as $M+L$ of the same section. The sagittal anterior diameter (A) was defined as the distance from the central axis to the anterior cortex of the sagittal plane, while the sagittal posterior diameter $(P)$ was defined as the distance from the central axis to the posterior cortex of the sagittal plane. The sagittal anterior-posterior parameter (AP) was defined as $A+P$ of the same section. The longest diameter (LD) was defined as the longest distance between any two points in the medial cortex. The vertical line of the LD (VL) was defined as the longest perpendicular line of the LD of the medullary cavity. The sectional area of the medullary cavity (SA) was also measured (Fig. 3).

(2) Measurement of non-horizontal plane parameters of the proximal femoral medullary cavity, including the offset, neck-shaft angle (NSA), anteversion angle, femoral head diameter, femoral head height, and femoral length (Fig. 2). 
(3) Calculated value of the proximal femoral medullary cavity:

$$
\mathrm{CFI60}=\frac{\mathrm{T}-20}{\mathrm{~T}+60}
$$

\subsection{Statistical analysis}

The coordinate system of the images was reconstructed by the same physician (R.Z.) for all participants. To ensure the repeatability of the establishment of the coordinate system, the coordinate system was reestablished after an interval of 1 month, and the angle between the coronal axes was measured. To ensure the reliability of the measurements of the medullary cavity parameters, all parameters were measured twice by two physicians (R.Z. and H.C.) at 1-month intervals. The intra-group correlation coefficient (ICC) was used to evaluate the measurement reliability as poor (ICC $0.00-0.20$ ), fair (ICC $0.21-$ 0.40), moderate (ICC 0.41-0.60), good (ICC 0.61-0.80), and excellent (ICC 0.81-1.00).

The Shapiro-Wilk test was used to test for distribution normality. Normally distributed data were presented as the mean and standard deviation, and the Student's t-test was used to test for significant differences. Abnormally distributed data or normally distributed data with different variances were presented as the median and quartiles, and the Mann-Whitney test was used to test for significant differences. The Pearson correlation analysis and partial correlation analysis were used to determine the relationships between anatomical parameters. A P value of $<0.05$ was considered statistically significant.

\section{Results}

The variation of the angle between the coronal axes of the coordinate system was less than $2^{\circ}$, and the ICC of the various indicators varied from 0.83 to 0.94 , which indicated that the measurement results were reliable. The cross-sectional parameters of the femoral medullary cavity ( $M L, M, L, A P, A$, and $P$ ) were normally distributed, and were tested by the Student's t-test. The data of the LD and the VL of the medullary cavity were not normally distributed, and were evaluated with the Mann-Whitney test.

The average femoral length was $382.0 \pm 27.9 \mathrm{~mm}$, and the average femoral anteversion angle was $14.2 \pm$ $8.0^{\circ}$. The proximal femoral canal parameters of the overall sample are detailed in Table 1, Table 4, and Fig. 4. 
Table 1

Coronal/Sagittal Diameters of the Femoral Medullary Cavity

\begin{tabular}{|c|c|c|c|c|c|c|c|}
\hline & ML & $M$ & $\mathbf{L}$ & AP & $A$ & $\mathbf{P}$ & SA \\
\hline T-25 & $\begin{array}{l}47.53 \pm \\
5.35\end{array}$ & $\begin{array}{l}29.36 \pm \\
4.29\end{array}$ & $\begin{array}{l}18.18 \pm \\
3.17\end{array}$ & $\begin{array}{l}32.14 \pm \\
4.66\end{array}$ & $\begin{array}{l}21.16 \pm \\
3.42\end{array}$ & $\begin{array}{l}10.98 \pm \\
4.55\end{array}$ & $\begin{array}{l}1194.61 \pm \\
234.90\end{array}$ \\
\hline $\mathrm{T}-20$ & $\begin{array}{l}44.70 \pm \\
5.11\end{array}$ & $\begin{array}{l}26.64 \pm \\
4.07\end{array}$ & $\begin{array}{l}18.06 \pm \\
3.92\end{array}$ & $\begin{array}{l}32.55 \pm \\
3.74\end{array}$ & $\begin{array}{l}19.15 \pm \\
3.49\end{array}$ & $\begin{array}{l}13.39 \pm \\
3.58\end{array}$ & $\begin{array}{l}1117.00 \pm \\
223.58\end{array}$ \\
\hline $\mathrm{T}-15$ & $\begin{array}{l}39.76 \pm \\
5.38\end{array}$ & $\begin{array}{l}22.83 \pm \\
4.32\end{array}$ & $\begin{array}{l}16.93 \pm \\
2.65\end{array}$ & $\begin{array}{l}25.84 \pm \\
4.09\end{array}$ & $\begin{array}{l}17.00 \pm \\
3.17\end{array}$ & $\begin{array}{l}8.45 \pm \\
3.42\end{array}$ & $\begin{array}{l}809.70 \pm \\
179.31\end{array}$ \\
\hline $\mathrm{T}-10$ & $\begin{array}{l}35.32 \pm \\
4.28\end{array}$ & $\begin{array}{l}19.71 \pm \\
3.00\end{array}$ & $\begin{array}{l}15.59 \pm \\
2.44\end{array}$ & $\begin{array}{l}23.98 \pm \\
5.71\end{array}$ & $\begin{array}{l}15.51 \pm \\
3.70\end{array}$ & $\begin{array}{l}8.00 \pm \\
3.08\end{array}$ & $\begin{array}{l}654.28 \pm \\
153.88\end{array}$ \\
\hline $\mathrm{T}-5$ & $\begin{array}{l}31.21 \pm \\
3.97\end{array}$ & $\begin{array}{l}16.91 \pm \\
2.66\end{array}$ & $\begin{array}{l}14.29 \pm \\
2.17\end{array}$ & $\begin{array}{l}22.64 \pm \\
3.93\end{array}$ & $\begin{array}{l}13.86 \pm \\
2.48\end{array}$ & $\begin{array}{l}8.40 \pm \\
2.68\end{array}$ & $\begin{array}{l}535.67 \pm \\
136.97\end{array}$ \\
\hline$T-0$ & $\begin{array}{l}27.41 \pm \\
3.80\end{array}$ & $\begin{array}{l}14.48 \pm \\
2.47\end{array}$ & $\begin{array}{l}12.93 \pm \\
2.10\end{array}$ & $\begin{array}{l}20.29 \pm \\
3.45\end{array}$ & $\begin{array}{l}12.41 \pm \\
2.17\end{array}$ & $\begin{array}{l}7.74 \pm \\
2.42\end{array}$ & $\begin{array}{l}443.78 \pm \\
120.24\end{array}$ \\
\hline $\begin{array}{l}T+ \\
5\end{array}$ & $\begin{array}{l}24.67 \pm \\
3.68\end{array}$ & $\begin{array}{l}12.92 \pm \\
2.28\end{array}$ & $\begin{array}{l}11.75 \pm \\
1.88\end{array}$ & $\begin{array}{l}19.71 \pm \\
4.01\end{array}$ & $\begin{array}{l}11.56 \pm \\
2.19\end{array}$ & $\begin{array}{l}7.76 \pm \\
2.41\end{array}$ & $\begin{array}{l}375.15 \pm \\
118.20\end{array}$ \\
\hline $\begin{array}{l}T+ \\
10\end{array}$ & $\begin{array}{l}22.34 \pm \\
3.25\end{array}$ & $\begin{array}{l}11.52+ \\
1.93\end{array}$ & $\begin{array}{l}10.82 \pm \\
1.68\end{array}$ & $\begin{array}{l}18.22 \pm \\
2.52\end{array}$ & $\begin{array}{l}10.78 \pm \\
1.79\end{array}$ & $\begin{array}{l}7.44 \pm \\
1.65\end{array}$ & $\begin{array}{l}309.38 \pm \\
84.01\end{array}$ \\
\hline $\begin{array}{l}T+ \\
15\end{array}$ & $\begin{array}{l}20.41 \pm \\
3.26\end{array}$ & $\begin{array}{l}10.52+ \\
2.12\end{array}$ & $\begin{array}{l}9.89 \pm \\
1.53\end{array}$ & $\begin{array}{l}17.71 \pm \\
2.51\end{array}$ & $\begin{array}{l}10.09 \pm \\
1.75\end{array}$ & $\begin{array}{l}7.57 \pm \\
1.75\end{array}$ & $\begin{array}{l}273.55 \pm \\
79.83\end{array}$ \\
\hline $\begin{array}{l}\mathrm{T}+ \\
20\end{array}$ & $\begin{array}{l}18.92 \pm \\
2.78\end{array}$ & $\begin{array}{l}9.71 \pm \\
1.69\end{array}$ & $\begin{array}{l}9.21 \pm \\
1.40\end{array}$ & $\begin{array}{l}17.09 \pm \\
2.18\end{array}$ & $\begin{array}{l}9.44 \pm \\
1.61\end{array}$ & $\begin{array}{l}7.65 \pm \\
1.30\end{array}$ & $\begin{array}{l}268.02 \pm \\
80.39\end{array}$ \\
\hline $\begin{array}{l}\mathrm{T}+ \\
30\end{array}$ & $\begin{array}{l}16.77 \pm \\
2.52\end{array}$ & $\begin{array}{l}8.46 \pm \\
1.48\end{array}$ & $\begin{array}{l}8.31 \pm \\
1.25\end{array}$ & $\begin{array}{l}15.86 \pm \\
2.78\end{array}$ & $\begin{array}{l}8.40 \pm \\
1.70\end{array}$ & $\begin{array}{l}7.46 \pm \\
1.42\end{array}$ & $\begin{array}{l}203.06 \pm \\
63.66\end{array}$ \\
\hline $\begin{array}{l}T+ \\
40\end{array}$ & $\begin{array}{l}15.34 \pm \\
2.28\end{array}$ & $\begin{array}{l}7.63 \pm \\
1.33\end{array}$ & $\begin{array}{l}7.71 \pm \\
1.12\end{array}$ & $\begin{array}{l}14.33 \pm \\
2.49\end{array}$ & $\begin{array}{l}7.84 \pm \\
1.28\end{array}$ & $\begin{array}{l}6.49 \pm \\
1.48\end{array}$ & $\begin{array}{l}166.46 \pm \\
52.97\end{array}$ \\
\hline $\begin{array}{l}\mathrm{T}+ \\
50\end{array}$ & $\begin{array}{l}14.35 \pm \\
2.10\end{array}$ & $\begin{array}{l}7.01 \pm \\
1.22\end{array}$ & $\begin{array}{l}7.34 \pm \\
1.04\end{array}$ & $\begin{array}{l}13.39 \pm \\
2.46\end{array}$ & $\begin{array}{l}7.36 \pm \\
1.39\end{array}$ & $\begin{array}{l}6.03 \pm \\
1.35\end{array}$ & $\begin{array}{l}145.64 \pm \\
48.40\end{array}$ \\
\hline $\begin{array}{l}T+ \\
60\end{array}$ & $\begin{array}{l}15.14 \pm \\
14.80\end{array}$ & $\begin{array}{l}6.67 \pm \\
1.26\end{array}$ & $\begin{array}{l}6.98 \pm \\
1.01\end{array}$ & $\begin{array}{l}13.07 \pm \\
252\end{array}$ & $\begin{array}{l}7.25 \pm \\
1.34\end{array}$ & $\begin{array}{l}5.83 \pm \\
1.32\end{array}$ & $\begin{array}{l}134.83 \pm \\
45.03\end{array}$ \\
\hline
\end{tabular}

ML: coronal medial-lateral diameter; M: coronal medial diameter; L: coronal lateral diameter; AP: sagittal anterior-posterior diameter; A: sagittal anterior diameter; P: sagittal posterior diameter; SA: sectional area

Most cross-sectional parameters significantly differed between men and women, with the mean medullary cavity being wider in men than women (Table 2, Table 3, Figure. 5). Except for the femoral length, there was no significant difference between sexes in the parameters of the non-horizontal plane of the femoral medullary cavity and the CFI60 (Table 4, Table 5). 
Table 2

Sex Comparisons of the Coronal Medial-Lateral Diameter Parameters of the Proximal Femoral Medullary Cavity

\begin{tabular}{|c|c|c|c|c|c|c|c|c|c|}
\hline ML & Male & Female & $P$ & Male(M) & Female(M) & $P$ & Male(L) & Female(L) & $\begin{array}{l}P \\
\text { value }\end{array}$ \\
\hline $\begin{array}{l}\mathrm{T}- \\
25\end{array}$ & $\begin{array}{l}48.39 \\
\pm 5.59\end{array}$ & $\begin{array}{l}47.04 \\
\pm 5.19\end{array}$ & 0.000 & $\begin{array}{l}29.72 \pm \\
4.54\end{array}$ & $\begin{array}{l}29.15 \pm \\
4.17\end{array}$ & 0.012 & $\begin{array}{l}18.68 \pm \\
3.26\end{array}$ & $\begin{array}{l}17.89 \pm \\
3.10\end{array}$ & 0.000 \\
\hline $\begin{array}{l}\mathrm{T}- \\
20\end{array}$ & $\begin{array}{l}45.16 \\
\pm 5.48\end{array}$ & $\begin{array}{l}44.44 \\
\pm 4.91\end{array}$ & 0.000 & $\begin{array}{l}26.65 \pm \\
4.23\end{array}$ & $\begin{array}{l}26.63 \pm \\
4.01\end{array}$ & 0.018 & $\begin{array}{l}18.50 \pm \\
3.16\end{array}$ & $\begin{array}{l}17.81 \pm \\
2.78\end{array}$ & 0.000 \\
\hline $\begin{array}{l}\mathrm{T}- \\
15\end{array}$ & $\begin{array}{l}40.27 \\
\pm 4.72\end{array}$ & $\begin{array}{l}39.46 \\
\pm 5.74\end{array}$ & 0.000 & $\begin{array}{l}23.00 \pm \\
3.58\end{array}$ & $\begin{array}{l}22.73 \pm \\
4.72\end{array}$ & 0.049 & $\begin{array}{l}17.27 \pm \\
2.77\end{array}$ & $\begin{array}{l}16.73 \pm \\
2.58\end{array}$ & 0.000 \\
\hline $\begin{array}{l}\mathrm{T}- \\
10\end{array}$ & $\begin{array}{l}35.59 \\
\pm 4.56\end{array}$ & $\begin{array}{l}35.17 \\
\pm 4.15\end{array}$ & 0.000 & $\begin{array}{l}19.69 \pm \\
3.12\end{array}$ & $\begin{array}{l}19.72 \pm \\
2.96\end{array}$ & 0.031 & $\begin{array}{l}15.90 \pm \\
2.59\end{array}$ & $\begin{array}{l}15.42 \pm \\
2.35\end{array}$ & 0.000 \\
\hline T-5 & $\begin{array}{l}31.49 \\
\pm 4.38\end{array}$ & $\begin{array}{l}31.05 \\
\pm 3.75\end{array}$ & 0.000 & $\begin{array}{l}16.80 \pm \\
2.82\end{array}$ & $\begin{array}{l}16.97 \pm \\
2.58\end{array}$ & 0.030 & $\begin{array}{l}14.69 \pm \\
2.38\end{array}$ & $\begin{array}{l}14.07 \pm \\
2.02\end{array}$ & 0.001 \\
\hline $\mathrm{T}-0$ & $\begin{array}{l}27.70 \\
\pm 4.51\end{array}$ & $\begin{array}{l}27.24 \\
\pm 3.36\end{array}$ & 0.001 & $\begin{array}{l}14.51 \pm \\
2.73\end{array}$ & $\begin{array}{l}14.46 \pm \\
2.33\end{array}$ & 0.040 & $\begin{array}{l}13.20 \pm \\
2.38\end{array}$ & $\begin{array}{l}12.78 \pm \\
1.93\end{array}$ & 0.001 \\
\hline $\begin{array}{l}\mathrm{T} \\
+5\end{array}$ & $\begin{array}{l}25.10 \\
\pm 4.16\end{array}$ & $\begin{array}{l}24.42 \\
\pm 3.38\end{array}$ & 0.000 & $\begin{array}{l}13.07 \pm \\
2.56\end{array}$ & $\begin{array}{l}12.83 \pm \\
2.11\end{array}$ & 0.001 & $\begin{array}{l}12.03 \pm \\
2.02\end{array}$ & $\begin{array}{l}11.59 \pm \\
1.80\end{array}$ & 0.002 \\
\hline $\begin{array}{l}\mathrm{T} \\
+ \\
10\end{array}$ & $\begin{array}{l}22.67 \\
\pm 3.69\end{array}$ & $\begin{array}{l}22.15 \\
\pm 2.99\end{array}$ & 0.000 & $\begin{array}{l}11.67 \pm \\
2.21\end{array}$ & $\begin{array}{l}11.43 \pm \\
1.76\end{array}$ & 0.000 & $\begin{array}{l}10.99 \pm \\
1.80\end{array}$ & $\begin{array}{l}10.73 \pm \\
1.62\end{array}$ & 0.002 \\
\hline $\begin{array}{l}\mathrm{T} \\
+ \\
15\end{array}$ & $\begin{array}{l}20.81 \\
\pm 3.62\end{array}$ & $\begin{array}{l}20.18 \\
\pm 3.04\end{array}$ & 0.000 & $\begin{array}{l}10.77 \pm \\
2.19\end{array}$ & $\begin{array}{l}10.37 \pm \\
2.09\end{array}$ & 0.000 & $\begin{array}{l}10.04 \pm \\
1.67\end{array}$ & $\begin{array}{l}9.81 \pm \\
1.45\end{array}$ & 0.001 \\
\hline $\begin{array}{l}T \\
+ \\
20\end{array}$ & $\begin{array}{l}19.01 \\
\pm 3.07\end{array}$ & $\begin{array}{l}18.87 \\
\pm 2.63\end{array}$ & 0.000 & $\begin{array}{l}9.80 \pm \\
1.86\end{array}$ & $9.65 \pm 1.60$ & 0.000 & $\begin{array}{l}9.21 \pm \\
1.49\end{array}$ & $\begin{array}{l}9.21 \pm \\
1.35\end{array}$ & 0.001 \\
\hline $\begin{array}{l}\mathrm{T} \\
+ \\
30\end{array}$ & $\begin{array}{l}16.80 \\
\pm 2.65\end{array}$ & $\begin{array}{l}16.75 \\
\pm 2.46\end{array}$ & 0.000 & $\begin{array}{l}8.51 \pm \\
1.55\end{array}$ & $8.43 \pm 1.45$ & 0.000 & $\begin{array}{l}8.29 \pm \\
1.28\end{array}$ & $\begin{array}{l}8.32 \pm \\
1.25\end{array}$ & 0.000 \\
\hline $\begin{array}{l}\mathrm{T} \\
+ \\
40\end{array}$ & $\begin{array}{l}15.32 \\
\pm 2.49\end{array}$ & $\begin{array}{l}15.35 \\
\pm 2.17\end{array}$ & 0.000 & $\begin{array}{l}7.61 \pm \\
1.46\end{array}$ & $7.64 \pm 1.26$ & 0.000 & $\begin{array}{l}7.72 \pm \\
1.16\end{array}$ & $\begin{array}{l}7.70 \pm \\
1.11\end{array}$ & 0.001 \\
\hline $\begin{array}{l}\mathrm{T} \\
+ \\
50\end{array}$ & $\begin{array}{l}14.35 \\
\pm 2.24\end{array}$ & $\begin{array}{l}14.35 \\
\pm 2.04\end{array}$ & 0.002 & $\begin{array}{l}6.98 \pm \\
1.36\end{array}$ & $7.03 \pm 1.15$ & 0.002 & $\begin{array}{l}7.37 \pm \\
1.03\end{array}$ & $\begin{array}{l}7.32 \pm \\
1.05\end{array}$ & 0.006 \\
\hline $\begin{array}{l}\mathrm{T} \\
+ \\
60\end{array}$ & $\begin{array}{l}13.57 \\
\pm 2.19\end{array}$ & $\begin{array}{l}13.68 \\
\pm 2.08\end{array}$ & 0.001 & $\begin{array}{l}6.66 \pm \\
1.24\end{array}$ & $6.67 \pm 1.28$ & 0.014 & $\begin{array}{l}6.91 \pm \\
1.05\end{array}$ & $\begin{array}{l}7.01 \pm \\
0.99\end{array}$ & 0.000 \\
\hline
\end{tabular}


Table 3

Sex Comparisons of the Sagittal Anterior-Posterior Diameter Parameters of the Proximal Femoral Medullary Cavity

\begin{tabular}{|c|c|c|c|c|c|c|c|c|c|}
\hline AP & Male & Female & $P$ & Male(A) & Female(A) & $P$ & Male(P) & Female(P) & $\begin{array}{l}P \\
\text { value }\end{array}$ \\
\hline $\begin{array}{l}\mathrm{T}- \\
25\end{array}$ & $\begin{array}{l}32.58 \\
\pm 4.45\end{array}$ & $\begin{array}{l}31.89 \pm \\
4.80\end{array}$ & 0.003 & $\begin{array}{l}21.08 \pm \\
3.20\end{array}$ & $\begin{array}{l}21.21 \pm \\
3.57\end{array}$ & 0.732 & $\begin{array}{l}11.50 \pm \\
3.74\end{array}$ & $\begin{array}{l}10.68 \pm \\
4.97\end{array}$ & 0.000 \\
\hline $\begin{array}{l}\mathrm{T}- \\
20\end{array}$ & $\begin{array}{l}32.53 \\
\pm 4.17\end{array}$ & $\begin{array}{l}32.56 \pm \\
3.51\end{array}$ & 0.007 & $\begin{array}{l}18.98 \pm \\
3.31\end{array}$ & $\begin{array}{l}19.26 \pm \\
3.61\end{array}$ & 0.838 & $\begin{array}{l}13.55 \pm \\
3.31\end{array}$ & $\begin{array}{l}13.30 \pm \\
3.75\end{array}$ & 0.000 \\
\hline $\begin{array}{l}\text { T- } \\
15\end{array}$ & $\begin{array}{l}26.05 \\
\pm 4.07\end{array}$ & $\begin{array}{l}25.72 \pm \\
4.13\end{array}$ & 0.069 & $\begin{array}{l}17.00 \pm \\
3.12\end{array}$ & $\begin{array}{l}17.00 \pm \\
3.23\end{array}$ & 0.641 & $\begin{array}{l}8.56 \pm \\
3.38\end{array}$ & $\begin{array}{l}8.38 \pm \\
3.47\end{array}$ & 0.145 \\
\hline $\begin{array}{l}\text { T- } \\
10\end{array}$ & $\begin{array}{l}24.65 \\
\pm 8.21\end{array}$ & $\begin{array}{l}23.60 \pm \\
3.61\end{array}$ & 0.028 & $\begin{array}{l}15.80 \pm \\
5.02\end{array}$ & $\begin{array}{l}15.35 \pm \\
2.70\end{array}$ & 0.507 & $\begin{array}{l}8.11 \pm \\
2.81\end{array}$ & $\begin{array}{l}7.94 \pm \\
3.25\end{array}$ & 0.028 \\
\hline T-5 & $\begin{array}{l}22.52 \\
\pm 4.18\end{array}$ & $\begin{array}{l}22.71 \pm \\
3.81\end{array}$ & 0.043 & $\begin{array}{l}13.75 \pm \\
2.65\end{array}$ & $\begin{array}{l}13.92 \pm \\
2.40\end{array}$ & 0.313 & $\begin{array}{l}8.30 \pm \\
2.44\end{array}$ & $\begin{array}{l}8.46 \pm \\
2.82\end{array}$ & 0.086 \\
\hline $\mathrm{T}-0$ & $\begin{array}{l}20.20 \\
\pm 2.98\end{array}$ & $\begin{array}{l}20.35 \pm \\
3.72\end{array}$ & 0.010 & $\begin{array}{l}12.33 \pm \\
2.51\end{array}$ & $\begin{array}{l}12.46 \pm \\
1.98\end{array}$ & 0.195 & $\begin{array}{l}7.87 \pm \\
2.19\end{array}$ & $\begin{array}{l}7.67 \pm \\
2.55\end{array}$ & 0.001 \\
\hline $\begin{array}{l}\mathrm{T} \\
+5\end{array}$ & $\begin{array}{l}20.15 \\
\pm 4.77\end{array}$ & $\begin{array}{l}19.45 \pm \\
3.51\end{array}$ & 0.013 & $\begin{array}{l}11.61 \pm \\
2.23\end{array}$ & $\begin{array}{l}11.52 \pm \\
2.18\end{array}$ & 0.190 & $\begin{array}{l}8.06 \pm \\
2.61\end{array}$ & $\begin{array}{l}7.60 \pm \\
2.28\end{array}$ & 0.003 \\
\hline $\begin{array}{l}\mathrm{T} \\
+ \\
10\end{array}$ & $\begin{array}{l}18.18 \\
\pm 2.51\end{array}$ & $\begin{array}{l}18.24 \pm \\
2.55\end{array}$ & 0.003 & $\begin{array}{l}10.66 \pm \\
2.11\end{array}$ & $\begin{array}{l}10.84 \pm \\
1.60\end{array}$ & 0.068 & $\begin{array}{l}7.52 \pm \\
1.51\end{array}$ & $\begin{array}{l}7.40 \pm \\
1.74\end{array}$ & 0.005 \\
\hline $\begin{array}{l}\mathrm{T} \\
+ \\
15\end{array}$ & $\begin{array}{l}17.43 \\
\pm 2.49\end{array}$ & $\begin{array}{l}17.87 \pm \\
2.53\end{array}$ & 0.002 & $\begin{array}{l}10.03 \pm \\
2.05\end{array}$ & $\begin{array}{l}10.13 \pm \\
1.56\end{array}$ & 0.092 & $\begin{array}{l}7.34 \pm \\
1.49\end{array}$ & $\begin{array}{l}7.70 \pm \\
1.88\end{array}$ & 0.006 \\
\hline $\begin{array}{l}\mathrm{T} \\
+ \\
20\end{array}$ & $\begin{array}{l}17.12 \\
\pm 2.11\end{array}$ & $\begin{array}{l}17.07 \pm \\
2.24\end{array}$ & 0.001 & $\begin{array}{l}9.43 \pm \\
1.95\end{array}$ & $\begin{array}{l}9.45 \pm \\
1.39\end{array}$ & 0.027 & $\begin{array}{l}7.70 \pm \\
1.19\end{array}$ & $\begin{array}{l}7.62 \pm \\
1.36\end{array}$ & 0.002 \\
\hline $\begin{array}{l}T \\
+ \\
30\end{array}$ & $\begin{array}{l}15.76 \\
\pm 2.92\end{array}$ & $\begin{array}{l}15.92 \pm \\
2.71\end{array}$ & 0.002 & $\begin{array}{l}8.35 \pm \\
1.91\end{array}$ & $\begin{array}{l}8.43 \pm \\
1.59\end{array}$ & 0.053 & $\begin{array}{l}7.41 \pm \\
1.30\end{array}$ & $\begin{array}{l}7.48 \pm \\
1.50\end{array}$ & 0.000 \\
\hline $\begin{array}{l}\mathrm{T} \\
+ \\
40\end{array}$ & $\begin{array}{l}14.24 \\
\pm 2.61\end{array}$ & $\begin{array}{l}14.39 \pm \\
2.43\end{array}$ & 0.000 & $\begin{array}{l}7.75 \pm \\
1.47\end{array}$ & $\begin{array}{l}7.90 \pm \\
1.16\end{array}$ & 0.012 & $\begin{array}{l}6.49 \pm \\
1.48\end{array}$ & $\begin{array}{l}6.49 \pm \\
1.50\end{array}$ & 0.000 \\
\hline $\begin{array}{l}\mathrm{T} \\
+ \\
50\end{array}$ & $\begin{array}{l}13.05 \\
\pm 2.52\end{array}$ & $\begin{array}{l}13.59 \pm \\
2.42\end{array}$ & 0.002 & $\begin{array}{l}7.04 \pm \\
1.67\end{array}$ & $\begin{array}{l}7.54 \pm \\
1.18\end{array}$ & 0.017 & $\begin{array}{l}6.01 \pm \\
1.32\end{array}$ & $\begin{array}{l}6.05 \pm \\
1.38\end{array}$ & 0.000 \\
\hline $\begin{array}{l}T \\
+ \\
60\end{array}$ & $\begin{array}{l}12.91 \\
\pm 2.49\end{array}$ & $\begin{array}{l}13.16 \pm \\
2.55\end{array}$ & 0.000 & $\begin{array}{l}7.16 \pm \\
1.43\end{array}$ & $\begin{array}{l}7.29 \pm \\
1.29\end{array}$ & 0.003 & $\begin{array}{l}5.75 \pm \\
1.24\end{array}$ & $\begin{array}{l}5.87 \pm \\
1.38\end{array}$ & 0.000 \\
\hline
\end{tabular}


Table 4

Total Sample and Sex Comparisons of the Non-Horizontal Parameters of the Proximal Femoral Medullary Cavity

\begin{tabular}{|lllll|}
\hline & Total & Male 31 & Female 63 & P value \\
\hline Femur length & $381.98 \pm 27.91$ & $402.67 \pm 28.11$ & $371.80 \pm 21.61$ & 0.048 \\
\hline Anteversion & $14.16 \pm 8.02$ & $13.91 \pm 8.31$ & $14.29 \pm 7.94$ & 0.671 \\
\hline Offset & $40.02 \pm 4.41$ & $41.72 \pm 3.62$ & $39.18 \pm 4.55$ & 0.975 \\
\hline Femur height & $45.04 \pm 6.94$ & $46.00 \pm 6.43$ & $44.50 \pm 7.21$ & 0.293 \\
\hline Diameter of femoral head & $43.76 \pm 4.07$ & $43.64 \pm 5.20$ & $43.83 \pm 3.30$ & 0.765 \\
\hline Isthmus height & $117.61 \pm 20.44$ & $119.29 \pm 18.89$ & $116.66 \pm 21.37$ & 0.398 \\
\hline Neck-shaft angle & $129.30 \pm 5.28$ & $128.66 \pm 6.15$ & $129.61 \pm 4.82$ & 0.987 \\
\hline
\end{tabular}

Table 5

Total Sample and Sex Comparisons of the Parameters of the Proximal Femoral Medullary Cavity

\begin{tabular}{|c|c|c|c|c|}
\hline & Total & Male 31 & Female 63 & $\mathrm{P}$ of gender comparison \\
\hline ML-CFI60 & $3.31 \pm 0.62$ & $3.23 \pm 0.76$ & $3.35 \pm 0.54$ & 0.662 \\
\hline M-CFI60 & $4.14 \pm 1.07$ & $3.91 \pm 0.86$ & $4.25 \pm 1.14$ & 0.959 \\
\hline ML-CFI60 & $2.64 \pm 0.52$ & $2.79 \pm 0.53$ & $2.57 \pm 0.50$ & 0.368 \\
\hline AP-CFI60 & $2.55 \pm 0.44$ & $2.40 \pm 0.33$ & $2.63 \pm 0.46$ & 0.584 \\
\hline A-CFI60 & $2.72 \pm 0.70$ & $2.45 \pm 0.54$ & $2.86 \pm 0.73$ & 0.526 \\
\hline P-CFI60 & $2.35 \pm 0.62$ & $2.37 \pm 0.61$ & $2.34 \pm 0.62$ & 0.323 \\
\hline SA-CFI60 & $8.88 \pm 2.60$ & $8.46 \pm 2.24$ & $9.09 \pm 2.76$ & 0.293 \\
\hline \multicolumn{5}{|c|}{ 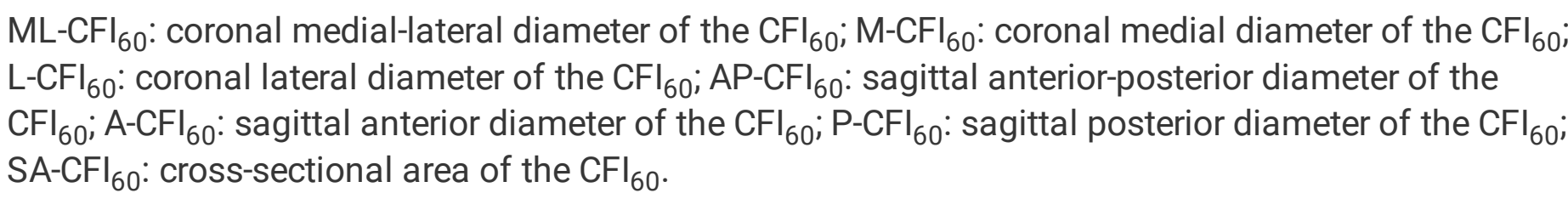 } \\
\hline
\end{tabular}

In the correlation analysis of the medullary cavity parameters, the length of the femur and the diameter of the femoral head were correlated with the ML, AP, and SA (Table 6). Age was negatively correlated with the ML of the CFI60 (ML-CFI60) and the L of the CFI60 (L-CFI60), i.e. the CFI60 decreased with age, mainly due to changes in the shape of the lateral medullary cavity. There was a positive correlation between the length of the femur, the diameter of the femoral head, and the parameters of the horizontal plane of the medullary cavity. The ML of the medullary cavity and the AP increased in tandem with increases in the length of the femur and the diameter of femoral head. There was a negative correlation between the 
NSA(neck shaft angel) and the ML-CFI60 and AP of the CFI60 (AP-CFI60), i.e. the CFI60 decreased in tandem with the increase in the NSA (Table 7). 
Table 6

Correlations between Cross-Sectional Parameters of the Femoral Medullary Cavity and Non-CrossSectional Parameters of the Femur

\begin{tabular}{|c|c|c|c|c|c|c|c|}
\hline & $\begin{array}{l}\text { Femur } \\
\text { length }\end{array}$ & Anteversion & Offset & $\begin{array}{l}\text { Femur } \\
\text { height }\end{array}$ & $\begin{array}{l}\text { Diameter of } \\
\text { femoral head }\end{array}$ & $\begin{array}{l}\text { Isthmus } \\
\text { height }\end{array}$ & $\begin{array}{l}\text { Neck- } \\
\text { shaft } \\
\text { angle }\end{array}$ \\
\hline $\begin{array}{l}\text { MLT- } \\
20\end{array}$ & $0.294^{\star \star}$ & 0.043 & $0.378^{\star \star}$ & -0.063 & $0.451^{\star *}$ & 0.182 & -0.118 \\
\hline $\begin{array}{l}\text { APT- } \\
20\end{array}$ & $0.412^{\star \star}$ & -0.219 & $0.281^{\star *}$ & 0.119 & $0.448^{\star *}$ & $0.258^{*}$ & 0.071 \\
\hline $\begin{array}{l}\text { SAT- } \\
20\end{array}$ & $0.448^{* *}$ & -0.077 & $0.341^{\star \star}$ & 0.034 & 0.591 ** & $0.282^{\star \star}$ & 0.041 \\
\hline $\begin{array}{l}\text { MLT- } \\
10\end{array}$ & 0.24 * & -0.027 & 0.179 & -0.166 & $0.395^{\star \star}$ & 0.192 & 0.053 \\
\hline $\begin{array}{l}\text { APT- } \\
10\end{array}$ & 0.184 & -0.041 & 0.149 & -0.024 & 0.148 & 0.097 & 0.026 \\
\hline $\begin{array}{l}\text { SAT- } \\
10\end{array}$ & $0.261^{\star}$ & -0.122 & 0.078 & -0.187 & $0.365^{\star \star}$ & 0.163 & 0.174 \\
\hline $\begin{array}{l}\text { MLT- } \\
0\end{array}$ & $0.309 * \star$ & 0.073 & 0.175 & -0.103 & $0.404^{\star *}$ & 0.200 & 0.095 \\
\hline APT-0 & 0.324 ** & -0.09 & $0.286^{\star \star}$ & -0.028 & 0.326 ** & 0.187 & 0.072 \\
\hline SAT-0 & $0.254^{\star}$ & -0.042 & 0.056 & -0.091 & $0.375^{\star \star}$ & 0.171 & 0.176 \\
\hline $\begin{array}{l}\text { MLT } \\
+20\end{array}$ & $0.450 * *$ & 0.059 & 0.116 & 0.162 & $0.483^{\star *}$ & $0.224^{\star}$ & $0.235^{\star}$ \\
\hline $\begin{array}{l}\text { APT } \\
+20\end{array}$ & 0.300 ** & -0.071 & 0.045 & 0.038 & $0.347 * \star$ & $0.354^{\star \star}$ & $0.219 *$ \\
\hline $\begin{array}{l}\text { SAT } \\
+20\end{array}$ & $0.403^{\star \star}$ & -0.023 & 0.038 & 0.165 & $0.485^{\star \star}$ & $0.222^{*}$ & $0.315^{\star \star}$ \\
\hline $\begin{array}{l}\text { MLT } \\
+60\end{array}$ & $0.412^{\star \star}$ & 0.138 & 0.11 & $0.311^{\star \star}$ & $0.423^{\star \star}$ & $0.258^{*}$ & $0.205^{\star}$ \\
\hline $\begin{array}{l}\text { APT } \\
+60\end{array}$ & $0.359 * \star$ & -0.153 & 0.029 & 0.360 ** & 0.496 ** & $0.235^{\star}$ & $0.270 \star \star$ \\
\hline $\begin{array}{l}\text { SAT } \\
+60\end{array}$ & 0.420 ** & -0.111 & -0.002 & $0.359 * \star$ & $0.495^{\star \star}$ & $0.283^{\star \star}$ & $0.359 \star \star$ \\
\hline \multicolumn{8}{|c|}{$\begin{array}{l}\text { MLT: coronal medial-lateral diameter of the corresponding level of the medullary cavity; APT: sagittal } \\
\text { anterior-posterior diameter of the corresponding level of the medullary cavity; SAT: corresponding } \\
\text { cross-sectional area of the medullary cavity. }\end{array}$} \\
\hline \multicolumn{8}{|c|}{${ }^{*} \mathrm{p}<0.05 ;{ }^{* *} \mathrm{p}<0.01$} \\
\hline
\end{tabular}


Table 7

Relationships between Age, Femoral Non-Medullary Interface Parameters, and Medullary Cross-Sectional Parameters

\begin{tabular}{|c|c|c|c|c|c|c|c|c|}
\hline & Age & $\begin{array}{l}\text { Femur } \\
\text { length }\end{array}$ & Anteversion & Offset & $\begin{array}{l}\text { Femur } \\
\text { height }\end{array}$ & $\begin{array}{l}\text { Diameter } \\
\text { of } \\
\text { femoral } \\
\text { head }\end{array}$ & $\begin{array}{l}\text { Isthmus } \\
\text { height }\end{array}$ & $\begin{array}{l}\text { Neck- } \\
\text { shaft } \\
\text { angle }\end{array}$ \\
\hline $\begin{array}{l}\mathrm{ML}- \\
\mathrm{CFI}_{60}\end{array}$ & 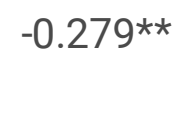 & $-0.250^{\star}$ & -0.086 & 0.159 & $-0.351 * \star$ & -0.131 & -0.08 & $-0.264^{\star}$ \\
\hline $\begin{array}{l}\mathrm{M}- \\
\mathrm{CFI}_{60}\end{array}$ & -0.076 & $0.225^{\star}$ & 0.071 & $0.321^{\star \star}$ & $-0.544^{\star \star}$ & -0.173 & -0.127 & -0.477 ** \\
\hline $\begin{array}{l}\mathrm{L}- \\
\mathrm{CFI} \\
60\end{array}$ & $-0.218^{*}$ & 0.088 & $-0.307 * \star$ & -0.170 & $0.252^{\star}$ & 0.176 & -0.024 & $0.258^{*}$ \\
\hline $\begin{array}{l}\mathrm{AP}- \\
\mathrm{CFI}_{60}\end{array}$ & -0.176 & -0.128 & 0.092 & 0.166 & $-0.342^{\star \star}$ & $-0.257^{*}$ & -0.122 & $-0.227^{\star}$ \\
\hline $\begin{array}{l}\text { A- } \\
\mathrm{CFI}_{60}\end{array}$ & -0.147 & -0.128 & 0.029 & 0.018 & $-0.552^{\star \star}$ & $-0.228^{*}$ & -0.075 & -0.130 \\
\hline $\begin{array}{l}\text { P- } \\
\mathrm{CFI}_{60}\end{array}$ & -0.064 & -0.175 & 0.08 & $0.263^{\star}$ & $0.256^{\star}$ & -0.077 & -0.089 & -0.183 \\
\hline $\begin{array}{l}\text { SA- } \\
\mathrm{CFI}_{60}\end{array}$ & -0.14 & -0.124 & 0.082 & $0.256^{\star}$ & $-0.384^{\star *}$ & -0.112 & -0.127 & $-0.328^{\star *}$ \\
\hline \multicolumn{9}{|c|}{ 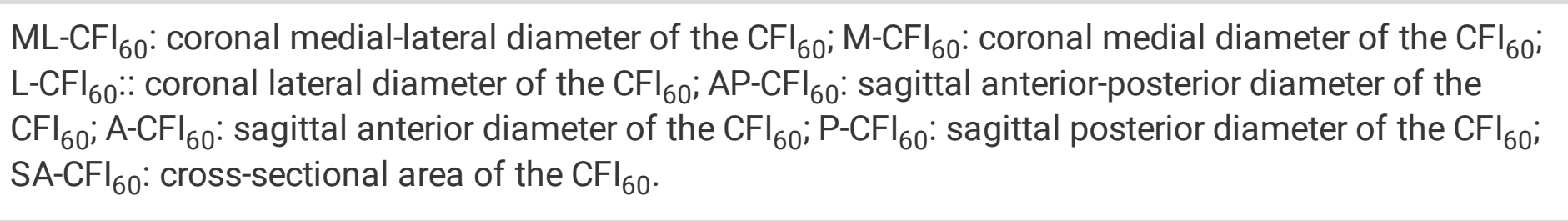 } \\
\hline${ }^{*} p<0$ & $\star \star p<0.0$ & & & & & & & \\
\hline
\end{tabular}

Table 8 shows the proximal femoral medullary cavity parameters in our study in comparison with those reported in previous studies. 
Table 8

Comparison of the Bone Morphology in Different Populations

\begin{tabular}{|c|c|c|c|c|c|}
\hline & This study & Yang et al.[8] & $\begin{array}{l}\text { Baharuddin et } \\
\text { al.[25] }\end{array}$ & Laine et al.[24] & $\begin{array}{l}\text { Sen et al. } \\
\text { [26] }\end{array}$ \\
\hline $\begin{array}{l}\text { Measurement } \\
\text { method }\end{array}$ & $\begin{array}{l}\text { 3D models } \\
\text { from CT }\end{array}$ & $\begin{array}{l}\text { 3D models } \\
\text { from CT }\end{array}$ & $\begin{array}{l}\text { 3D models } \\
\text { from CT }\end{array}$ & CT & $\mathrm{CT}$ \\
\hline Sample size & 63(94hip) & 80(160hip) & 60 & 50 & 50 \\
\hline Ethnicity & $\begin{array}{l}\text { Asian } \\
\text { (Chinese) }\end{array}$ & $\begin{array}{l}\text { Asian } \\
\text { (Chinese) }\end{array}$ & Asian (Malay) & $\begin{array}{l}\text { Caucasian } \\
\text { (Finnish) }\end{array}$ & Indian \\
\hline Offset & $40.02 \pm 4.41$ & $37.2 \pm 3.9 *$ & $30.35 \pm 42.6^{*}$ & - & $42 \pm 6^{*}$ \\
\hline $\begin{array}{l}\text { Neck-shaft } \\
\text { angle }\end{array}$ & $129.30 \pm 5.28$ & $130.2 \pm 4.3$ & $130.5 \pm 4.0$ & - & $132 \pm 6^{*}$ \\
\hline MLT-20 & $44.70 \pm 5.11$ & $41.69 \pm 4.43^{\star}$ & $44.1 \pm 4.6$ & $45.42 \pm 4.46$ & - \\
\hline APT-20 & $32.55 \pm 3.74$ & $31.00 \pm 3.29 *$ & $31.12 \pm 3.70 *$ & $31.39 \pm 3.45$ & - \\
\hline LDT-20 & $47.46 \pm 5.54$ & - & $46.21 \pm 4.63$ & $47.09 \pm 4.98$ & - \\
\hline MLT-0 & $27.41 \pm 3.80$ & $25.62 \pm 4.02^{\star}$ & $29.30 \pm 4.75^{\star}$ & $28.73 \pm 3.21^{*}$ & $\begin{array}{l}27.27 \pm \\
6.23\end{array}$ \\
\hline APT-0 & $20.29 \pm 3.45$ & $23.91 \pm 3.46^{*}$ & $23.56 \pm 3.08 *$ & $25.58 \pm 2.86^{\star}$ & $\begin{array}{l}23.44 \pm \\
3.78^{\star}\end{array}$ \\
\hline LDT-0 & $28.08 \pm 4.35$ & - & $38.16 \pm 5.12^{\star}$ & $31.01 \pm 3.65^{\star}$ & $\begin{array}{l}29.21 \pm \\
6.51\end{array}$ \\
\hline$M L T+20$ & $18.92 \pm 2.78$ & $19.78 \pm 2.43^{\star}$ & $18.45 \pm 2.93^{*}$ & $20.41 \pm 2.14 *$ & $\begin{array}{l}17.92 \pm \\
3.37\end{array}$ \\
\hline APT + 20 & $17.09 \pm 2.18$ & $17.50 \pm 3.05$ & $17.54 \pm 2.93$ & $20.71 \pm 2.47^{\star}$ & $\begin{array}{l}17.01 \pm \\
3.01\end{array}$ \\
\hline LDT + 20 & $20.27 \pm 3.06$ & - & $20.78 \pm 3.35$ & $23.19 \pm 2.74 *$ & $\begin{array}{l}18.71 \pm \\
3.92^{\star}\end{array}$ \\
\hline \multicolumn{6}{|c|}{$\begin{array}{l}\text { MLT: coronal medial-lateral diameter at the corresponding level of the medullary cavity; APT: sagittal } \\
\text { anterior-posterior diameter at the corresponding level of the medullary cavity; LDT: longest diameter of } \\
\text { the medullary cavity at the corresponding level. }\end{array}$} \\
\hline
\end{tabular}

\section{Discussion}

A variety of hip diseases are effectively treated by THA, which relieves hip pain and improves hip function. The cementless THA prothesis stem has good mid- and long-term survival [11-13]. Early and long-term stability after implantation depend on the achievement of a close fit between the stem and the proximal femoral medullary cavity; micromotion after implantation promotes fibrous tissue growth rather than osseointegration, and this leads to early postoperative failure $[14,15]$. 
Hip replacement surgery was introduced in the early 19th century, and has since continued to develop. With the continuous development of prosthetic design and the use of testing methods such as finite element analysis and photoelastic coating analysis, it has been proved that metaphyseal fixed prostheses are biomechanically sound and minimize stress shielding and disadvantageous bone remodeling $[16,17]$. Therefore, the use of short stems in primary THA is increasing, and has good clinical efficacy [18].

Radiographic measurements provide acceptable accuracy in measuring the shape of the proximal femoral medullary cavity, and radiography remains the most convenient method for assessing conventional THA. However, medullary measurements vary with leg rotation, which significantly alters the NSA and isthmus width [19]. In comparison with radiographic measurements, CT-based anatomical measurements have improved accuracy and reduced errors, as CT it is not affected by femoral rotation and the subjective determination of the bone threshold. Preoperative CT planning provides accurate anatomical information of the medullary cavity, and provides strong evidence for determining surgical goals and plans, especially in the preoperative design of custom prostheses [20].

In the process of CT scanning, the position of the patient cannot be completely controlled, even with the most rigid positioning, which often causes asymmetry of the pelvis and makes the calculations inaccurate. In addition, the traditional method of using the coronal or sagittal axis of the CT scan itself as the coordinate system does not coincide with the coordinate system corresponding to the implant placement during the operation. Therefore, we abandoned the use of the CT coordinate system as the sagittal and coronal planes. Furthermore, as the femur itself has an anterior arch and rotation, the axis of the femoral medullary canal is not straight, although the axis of the proximal femoral medullary cavity is usually approximated with a straight line.

During the establishment of the CT measurement coordinate system in the present study, we did not strictly use the coordinate system provided by the original CT scan and the "cortical-medullary cavity" for division and measurement. The following points were considered during the establishment of our methods of measurement.

1. 1. The plane passing through the center of the femoral head and the axis of the medullary cavity was used as the frontal plane to measure the shape of the medullary cavity and to guide the design of the prosthetic stem. This was done to restore the central position of the femoral head as much as possible.

2. 2. The femoral calcar is located in the posteromedial part of the junction between the femoral neck and the femoral shaft, deep in the lesser trochanter, and extends from the posterior side of the femoral neck to the longitudinal dense bone plate behind the lesser trochanter. CT imaging showed that the density of metaphyseal cancellous bone in the lesser trochanter and the proximal and posterior medullary cavity was lower than that of cortical bone, but higher than that of the medullary cavity, and the cancellous bone in this area was not removed by intraoperative reaming. The aim of analyzing the medullary cavity morphology was to aid in the design of femoral prostheses. The femoral stem is often press-fitted through the inside and outside, while its anterior-posterior diameter, 
especially the sagittal posterior diameter, does not contact the cortical bone. Therefore, to attain a better fitting stem design, this cancellous area should not be included in the medullary cavity measurements (yellow part in Fig. 2).

The measurement method used in the present study showed that there were still significant differences between the ML and LD and between the AP and VL (Table 9), but the degree of difference was not very large. Furthermore, the cross-sectional measurement shape tended to be elliptical, while threshold separation is strictly implemented in the measurement of the maximum diameter using Mimics software, which may be the reason for the significant differences between the ML and LD and between the AP and VL. In the present study, the medullary cavity cross-sectional parameters were measured, which avoids the measurement differences caused by the establishment of a different coordinate system, and is an effective supplement to the measurement of the medullary cavity coronal diameter and sagittal diameter [10]. 
Table 9

Differences between the coronal ML and LD and between the AP and VL

\begin{tabular}{|c|c|c|c|c|c|c|}
\hline & $\begin{array}{l}\text { ML [Median (Q1 } \\
\text { - Q3)] }\end{array}$ & $\begin{array}{l}\text { LD [Median (Q1 - } \\
\text { Q3)] }\end{array}$ & P & $\begin{array}{l}\text { AP [Median (Q1 } \\
\text { - Q3)] }\end{array}$ & $\begin{array}{l}\text { VL [Median (Q1 } \\
\text { - Q3)] }\end{array}$ & $\mathbf{P}$ \\
\hline $\begin{array}{l}T- \\
25\end{array}$ & $\begin{array}{l}46.78(43.59 \\
52.01)\end{array}$ & $\begin{array}{l}50.08(45.88 \\
54.44)\end{array}$ & 0.000 & $\begin{array}{l}31.58 \\
(29.57,34.41)\end{array}$ & $\begin{array}{l}32.33(28.65 \\
36.13)\end{array}$ & 0.421 \\
\hline $\begin{array}{l}T- \\
20\end{array}$ & $\begin{array}{l}44.55(40.51, \\
48.95)\end{array}$ & $\begin{array}{l}46.67(42.90 \\
51.74)\end{array}$ & 0.000 & $\begin{array}{l}31.85(30.58 \\
34.35)\end{array}$ & $\begin{array}{l}30.50 \\
(28.70,33.84)\end{array}$ & 0.000 \\
\hline $\begin{array}{l}\text { T- } \\
15\end{array}$ & $\begin{array}{l}39.95(35.99 \\
43.44)\end{array}$ & $\begin{array}{l}41.27(37.89 \\
45.31)\end{array}$ & 0.000 & $\begin{array}{l}25.37(22.74 \\
28.80)\end{array}$ & $\begin{array}{l}\text { 24.15 (22.21, } \\
26.75)\end{array}$ & 0.002 \\
\hline $\begin{array}{l}\text { T- } \\
10\end{array}$ & $\begin{array}{l}34.86(32.01 \\
38.22)\end{array}$ & $\begin{array}{l}36.09 \text { (32.48, } \\
40.15)\end{array}$ & 0.000 & $\begin{array}{l}22.86(20.71 \\
25.47)\end{array}$ & $\begin{array}{l}22.95(20.12 \\
25.10)\end{array}$ & 0.092 \\
\hline$T-5$ & $\begin{array}{l}30.72(28.36 \\
33.93)\end{array}$ & $\begin{array}{l}31.49(28.15 \\
34.56)\end{array}$ & 0.160 & $\begin{array}{l}21.89(20.26 \\
24.27)\end{array}$ & $\begin{array}{l}21.80(20.22 \\
23.59)\end{array}$ & 0.167 \\
\hline$T-0$ & $\begin{array}{l}27.23(24.74 \\
30.06)\end{array}$ & $\begin{array}{l}27.57(4.47 \\
31.09)\end{array}$ & 0.645 & $\begin{array}{l}19.60(17.72, \\
22.65)\end{array}$ & $\begin{array}{l}\text { 19.67 (17.75, } \\
22.56)\end{array}$ & 0.467 \\
\hline $\begin{array}{l}T+ \\
5\end{array}$ & $\begin{array}{l}24.65(21.75 \\
27.40)\end{array}$ & $\begin{array}{l}24.71(21.86 \\
27.84)\end{array}$ & 0.337 & $\begin{array}{l}\text { 18.81 (17.24, } \\
21.77)\end{array}$ & $\begin{array}{l}18.46(16.88 \\
20.53)\end{array}$ & 0.002 \\
\hline $\begin{array}{l}T+ \\
10\end{array}$ & $\begin{array}{l}22.08(19.72, \\
24.96)\end{array}$ & $\begin{array}{l}22.12(19.71, \\
24.56)\end{array}$ & 0.635 & $\begin{array}{l}18.24(16.43 \\
19.87)\end{array}$ & $\begin{array}{l}17.33(15.86 \\
19.09)\end{array}$ & 0.000 \\
\hline $\begin{array}{l}T+ \\
15\end{array}$ & $\begin{array}{l}19.99(18.09, \\
22.89)\end{array}$ & $\begin{array}{l}20.19(18.55 \\
22.93)\end{array}$ & 0.556 & $\begin{array}{l}17.54(15.86 \\
19.46)\end{array}$ & $\begin{array}{l}\text { 16.98 (15.02, } \\
19.00)\end{array}$ & 0.001 \\
\hline $\begin{array}{l}T+ \\
20\end{array}$ & $\begin{array}{l}18.83(16.61, \\
21.22)\end{array}$ & $\begin{array}{l}19.67(18.29 \\
22.15)\end{array}$ & 0.000 & $\begin{array}{l}17.04(15.38 \\
18.42)\end{array}$ & $\begin{array}{l}17.46(15.65 \\
19.90)\end{array}$ & 0.000 \\
\hline $\begin{array}{l}T+ \\
30\end{array}$ & $\begin{array}{l}\text { 16.53 (15.07, } \\
18.59)\end{array}$ & $\begin{array}{l}\text { 16.79 (15.66, } \\
19.63)\end{array}$ & 0.000 & $\begin{array}{l}\text { 15.31 (14.36, } \\
17.02)\end{array}$ & $\begin{array}{l}\text { 14.58 (13.10, } \\
16.48)\end{array}$ & 0.000 \\
\hline $\begin{array}{l}T+ \\
40\end{array}$ & $\begin{array}{l}\text { 15.08 (13.53, } \\
17.08)\end{array}$ & $\begin{array}{l}\text { 15.73 (14.55, } \\
17.31)\end{array}$ & 0.000 & $\begin{array}{l}13.78(12.51 \\
15.74)\end{array}$ & $\begin{array}{l}12.61(11.83 \\
14.77)\end{array}$ & 0.000 \\
\hline $\begin{array}{l}T+ \\
50\end{array}$ & $\begin{array}{l}13.99(12.58 \\
15.70)\end{array}$ & $\begin{array}{l}\text { 15.37 (13.93, } \\
16.64)\end{array}$ & 0.000 & $\begin{array}{l}13.02(11.75 \\
14.61)\end{array}$ & $\begin{array}{l}11.98(10.86 \\
13.47)\end{array}$ & 0.000 \\
\hline $\begin{array}{l}T+ \\
60\end{array}$ & $\begin{array}{l}13.26(12.33 \\
14.98)\end{array}$ & $\begin{array}{l}14.53(13.32 \\
16.50)\end{array}$ & 0.000 & $\begin{array}{l}12.57(11.50 \\
14.21)\end{array}$ & $\begin{array}{l}11.46(10.31 \\
12.53)\end{array}$ & 0.000 \\
\hline
\end{tabular}

ML: coronal medial-lateral diameter of the medullary cavity; LD: longest diameter of the medullary cavity; AP: sagittal anterior-posterior diameter of the medullary cavity; VL: vertical line of the longest diameter of the medullary cavity

The present study focused on the femoral medullary cavity from the level $25 \mathrm{~mm}$ proximal to $60 \mathrm{~mm}$ distal to the lesser trochanteric line, which does meet the needs of the medullary cavity design of shortstem prostheses. At present, the design concept of the cementless prosthetic stem is the internal and 
external press-fit fixation of the medullary cavity. Although the study of the 3D morphology of the medullary cavity using CT has gradually increased, most studies have focused on the medial and lateral morphology of the medullary cavity rather than the anterior-posterior diameter [5, 21]. The present study found that the medial-lateral morphology of the medullary cavity of the proximal femur had the following characteristics. 1 . The ML of the medullary cavity gradually narrowed from proximal to distal, and the trend of narrowing gradually decreased; this narrowing was less obvious at the levels of MLT +5 or MLT + 10. 2. $M$ was wider than $L$ in the metaphysis of the medullary cavity; the distal constriction was particularly obvious proximal to the MT +10 . The present study also evaluated the AP morphology of the medullary cavity, which revealed the following characteristics. 1 . The A of the medullary cavity gradually narrowed from the proximal to the distal end, and this narrowing was less obvious from AT + 5 or AT + 10 . 2. The PT-25 was smaller than PT-20, and tended to be straight from the level of PT-10 to PT +30 .

Our study showed that the $M L$ and $A P$ were not symmetrical at the reaming point. $M$ and $A$ were longer than $L$ and $P$, respectively, and the femoral osteotomy surface may have a smaller $S$ than the distal end. Under such circumstances, if the medullary cavity was opened at the midpoint, this would often lead to the reaming point being placed anteriorly and medially; thus, the selected reaming point should be as lateral and posterior as possible. Current stem designs have minimal width in the anterior-posterior dimensions, which are not connected to cortical bone; however, as stem designs become shorter and have more filling geometries within the anterior-posterior direction (fit and fill designs), a 3D model may be required. It may be optimal to set the AP of the metaphyseal stem asymmetrically to better match the medullary cavity and guide the placement of the prosthetic stem, especially in cases with excessive bowing of the femur.

Previous studies have reported differences in femoral medullary cavity anatomy between the sexes. Compared with females, males reportedly have a wider diameter of the femoral head, ML and AP of the medullary cavity, but a smaller CFI, indicating that the isthmus of the medullary cavity is wider in males than in females [8]. Another study reported that the width of the metaphysis of the medullary cavity is similar in males and females, but is wider in males at the diaphysis [10]; furthermore, the CFI60 in the coronal and sagittal planes is smaller in males than in females in those aged $<80$ years, but the CFI60 of women $>80$ years old is smaller than that of men $>80$ years old [10]. In our study, the average length of the femur was longer in men than women, while the mean medullary cavity was wider in women than men. However, there were no significant differences between sexes regarding offset, anteversion, NSA, and CFI60. This suggests that men and women can use the same brand of prosthesis, except that men require a larger stem. Previous research has shown that age affects the morphology of the femoral medullary cavity; with increasing age, the bone cortex becomes thinner, and the medullary cavity at the femoral diaphysis enlarges $[10,22,23]$. In our study, the ML of the CFI60 and L of the CFI60 decreased with age, suggesting a gradual transition of the medullary cavity morphology from a champagne glass shape to a chimney shape.

The morphology of the proximal end of the femoral medullary cavity is influenced by human ethnicity. One study found significant differences in medullary cavity morphology between Caucasian and 
Japanese and non-Japanese Asian women [5]; however, the femoral NSA was similar in all Asian races [5]. The proximal femoral medullary cavity of the present northern Chinese study population was similar to that described in a Caucasian population [24], but there were significant differences at the level of the lesser trochanteric line and distally, suggesting that the application of the same type of stem may cause distal implantation difficulties for Asians, and that Asians may require a finer distal stem than Caucasians. There were still differences between the present study population from northern China in comparison with a previous study population from southern China [8]. Therefore, even within the same race, the same implant may lead to unsatisfactory surgical results in the mismatched part of the population. Femoral medullary cavity morphology is determined by a series of genetic and environmental factors, such as ethnicity, sex, age, and lifestyle, which all contribute to the formation of a unique geometrical skeletal structure [9].

In the present study, the cross-sectional parameters of the medullary cavity were positively correlated with the femoral length and femoral head diameter, suggesting that the medullary cavity became wider as the height increased. Femoral head height was negatively correlated with the ML-CFI60, AP-CFI60, M of the CFI60, and A of the CFI60, and negatively correlated with the L-CFI60 and P of the CFI60. A negative correlation was also found between the NSA and the ML-CFI60 and AP-CFI60.

The present study had the following limitations. 1. The study only focused on the proximal femoral medullary cavity morphology of the northern Chinese population. Previous literature has demonstrated differences in medullary cavity morphology between Asian and Caucasian races [5]. However, the study has limited explanatory power regarding Caucasian medullary cavity morphology. 2 . The sample size was only 94 , and the oldest participant was only 68 years old. Thus, the medullary cavity morphology of the very elderly population was not evaluated. 3 . The present study participants were all healthy, and so the present results cannot be generalized to patients with diseases such as developmental hip dysplasia, ischemic necrosis of the femoral head epiphysis, and intracapsular/extracapsular deformity of the hip. However, this was not the aim of the present study. For patients with femoral deformity, a disease-specific bone morphology database should be established to aid in the design of prosthesis engagement at specific points and in 3D placement.

\section{Conclusions}

The measurement of morphological and anatomical data of the proximal femoral medullary cavity is important when formulating the procedures and evaluation guidelines for bone-to-prosthesis matching. The CFI is more greatly affected by age than by sex. Even if the prosthesis design is commercialized and non-personalized, individual variability should still be considered, especially in older adults or diseasespecific populations.

\section{Abbreviations}

THA, total hip arthroplasty 
$\mathrm{CFI}$, canal flare index

ICC, intra-group correlation coefficient

NSA, neck shaft angel

$\mathrm{L}$, coronal lateral diameter

$\mathrm{ML}$, the coronal medial-lateral diameter

A, the sagittal anterior diameter

P, the sagittal posterior diameter

$\mathrm{AP}$, the sagittal anterior-posterior parameter

LD, the longest diameter

$V L$, the vertical line of the LD

$\mathrm{M}$, coronal medial diameter

SA, sectional area

MLT, coronal medial-lateral diameter at the corresponding level of the medullary cavity

APT, sagittal anterior-posterior diameter at the corresponding level of the medullary cavity

LDT, longest diameter of the medullary cavity at the corresponding level

\section{Declarations}

\section{Ethics approval and consent to participate}

This project "Design verification and clinical application of individualized implant prosthesis" is the ethics statement of the ministry of Science and technology of China (no. 2016YFB1101501), which has been approved by the Ministry of science and technology of China, and passed the ethical review. The article" CT Measurement of Proximal Femoral Medullary Cavity Morphology in Healthy Subjects from Northern China" is a subproject, and is supported and approved by the ministry of Science and technology of China (no. 2016YFB1101501). The consent we obtained from study participants was written and shown in suppplemetary file.

\section{Consent for publication}

Not applicable. 


\section{Availability of data and materials}

All data generated or analysed during this study are included in this published article [and its supplementary information files].

\section{Competing interests}

The authors declare that they have no competing interests.

\section{Funding}

The authors thank the grant from the Ministry of Science and Technology of China (no. 2016YFB1101501). The funders had no role in study design, data collection and analysis, decision to publish or preparation of the manuscript.

\section{Authors' contributions}

RZ: data collection and management, data analysis, manuscript writing; HC: project development, manuscript editing; $\mathrm{HT}$ : data management, project development; KZ: data management, project development. All authors read and approved the final manuscript.

\section{Acknowledgment}

We acknowledge Kelly Zammit, BVSc, from Liwen Bianji, Edanz Editing China (www.liwenbianji.cn/ac), for editing the English text of a draft of this manuscript.

\section{References}

1. Learmonth ID, Young C, Rorabeck C. The operation of the century: total hip replacement. Lancet. 2007;370(9597):1508.

2. Massin P, Geais L, Astoin E, Simondi M, Lavaste F. The anatomic basis for the concept of lateralized femoral stems: a frontal plane radiographic study of the proximal femur. J Arthroplast. 2000;15(1):93.

3. Ishii S, Homma Y, Baba T, Ozaki Y, Matsumoto M, Kaneko K. Does the Canal Fill Ratio and Femoral Morphology of Asian Females Influence Early Radiographic Outcomes of Total Hip Arthroplasty With an Uncemented Proximally Coated, Tapered-Wedge Stem? J Arthroplast. 2016;31(7):1524.

4. Cooper HJ, Jacob AP, Rodriguez JA. Distal fixation of proximally coated tapered stems may predispose to a failure of osteointegration. J Arthroplast. 2011;26(6 Suppl):78.

5. Wuestemann T, Hoare SG, Petersik A, Hofstaetter B, Fehily M, Matsubara M, Markel DC. Bone morphology of the proximal femoral canal: ethnicity related differences and the influence on cementless tapered wedge stem designs. Hip international: the journal of clinical and experimental research on hip pathology and therapy: 1120700019895458, 2019. 
6. Zhao R, Cai H, Liu Y, Tian H, Zhang K, Liu Z. Risk Factors for Intraoperative Proximal Femoral Fracture During Primary Cementless THA. Orthopedics. 2017;40(2):e281.

7. Tang ZH, Yeoh CSN, Tan GMJ. Radiographic study of the proximal femur morphology of elderly patients with femoral neck fractures: is there a difference among ethnic groups? Singapore Med J. 2017;58(12):717.

8. Yang Z, Jian W, Li Z-h, Jun X, Liang Z, Ge Y. Shi Z-j. The geometry of the bone structure associated with total hip arthroplasty. PLoS One. 2014;9(3):e91058.

9. Noble PC, Alexander JW, Lindahl LJ, Yew DT, Granberry WM, Tullos HS. The anatomic basis of femoral component design. Clin Orthop Relat Res. 1988;235:148.

10. Boymans TA, Heyligers IC, Grimm B. The Morphology of the Proximal Femoral Canal Continues to Change in the Very Elderly: Implications for Total Hip Arthroplasty. J Arthroplast. 2015;30(12):2328.

11. Rorabeck $\mathrm{CH}$. Cementless femoral components should be made from cobalt chrome. Clin Orthop Relat Res. 1996;325:326.

12. Tostain O, Debuyzer E, Benad K, Putman S, Pierache A, Girard J, Pasquier G. Ten-year outcomes of cementless anatomical femoral implants after 3D computed tomography planning. Follow-up note. Orthopaedics \& traumatology, surgery \& research: OTSR 105(5): 937, 2019.

13. Cassidy RS, Oh S, Beverland DE. Guidelines for the follow-up of total hip arthroplasty: do they need to be revised? The bone \& joint journal 101-b(5): 536, 2019.

14. Lennon $A B$, Prendergast PJ. Evaluation of cement stresses in finite element analyses of cemented orthopaedic implants. J Biomech Eng. 2001;123(6):623.

15. Goodman SB. The effects of micromotion and particulate materials on tissue differentiation. Bone chamber studies in rabbits. Acta orthopaedica Scandinavica Supplementum. 1994;258:1.

16. Trieb K, Huber D, Sonntag R, Kretzer JP. Finite Element Analysis and Biomechanical Testing of the New MiniMIS Short Stem. Zeitschrift fur Orthopadie Unfallchirurgie. 2019;157(2):188.

17. Walker PS, Robertson DD. Design and fabrication of cementless hip stems. Clin Orthop Relat Res. 1988;235:25.

18. Liang H-D, Yang W-Y, Pan J-K, Huang H-T, Luo M-H, Zeng L-F, Liu J. Are short-stem prostheses superior to conventional stem prostheses in primary total hip arthroplasty? A systematic review and meta-analysis of randomised controlled trials. BMJ Open. 2018;8(9):e021649.

19. Rubin PJ, Leyvraz PF, Aubaniac JM, Argenson JN, Estève P, de Roguin B. The morphology of the proximal femur. A three-dimensional radiographic analysis. The Journal of bone joint surgery British volume. 1992;74(1):28.

20. Haddad FS, Garbuz DS, Duncan CP, Janzen DL, Munk PL. CT evaluation of periacetabular osteotomies. The Journal of bone joint surgery British volume. 2000;82(4):526.

21. Noble PC, Alexander JW, Lindahl LJ, Yew DT, Granberry WM, Tullos HS. The anatomic basis of femoral component design. Clin Orthop Relat Res. 1988;235:148. 
22. Casper DS, Kim GK, Parvizi J, Freeman TA. Morphology of the proximal femur differs widely with age and sex: relevance to design and selection of femoral prostheses. Journal of orthopaedic research: official publication of the Orthopaedic Research Society. 2012;30(7):1162.

23. Ruff CB, Hayes WC. Sex differences in age-related remodeling of the femur and tibia. Journal of orthopaedic research: official publication of the Orthopaedic Research Society. 1988;6(6):886.

24. Laine HJ, Lehto MU, Moilanen T. Diversity of proximal femoral medullary canal. J Arthroplast. 2000;15(1):86.

25. Baharuddin MY, Salleh S-H, Zulkifly AH, Lee MH, Mohd Noor A. Morphological study of the newly designed cementless femoral stem. Biomed Res Int 2014: 692328, 2014.

26. Sen RK, Tripathy SK, Kumar R, Kumar A, Dhatt S, Dhillon MS, Nagi ON, Gulati M. Proximal femoral medullary canal diameters in Indians: correlation between anatomic, radiographic, and computed tomographic measurements. J Orthop Surg. 2010;18(2):189.

\section{Figures}
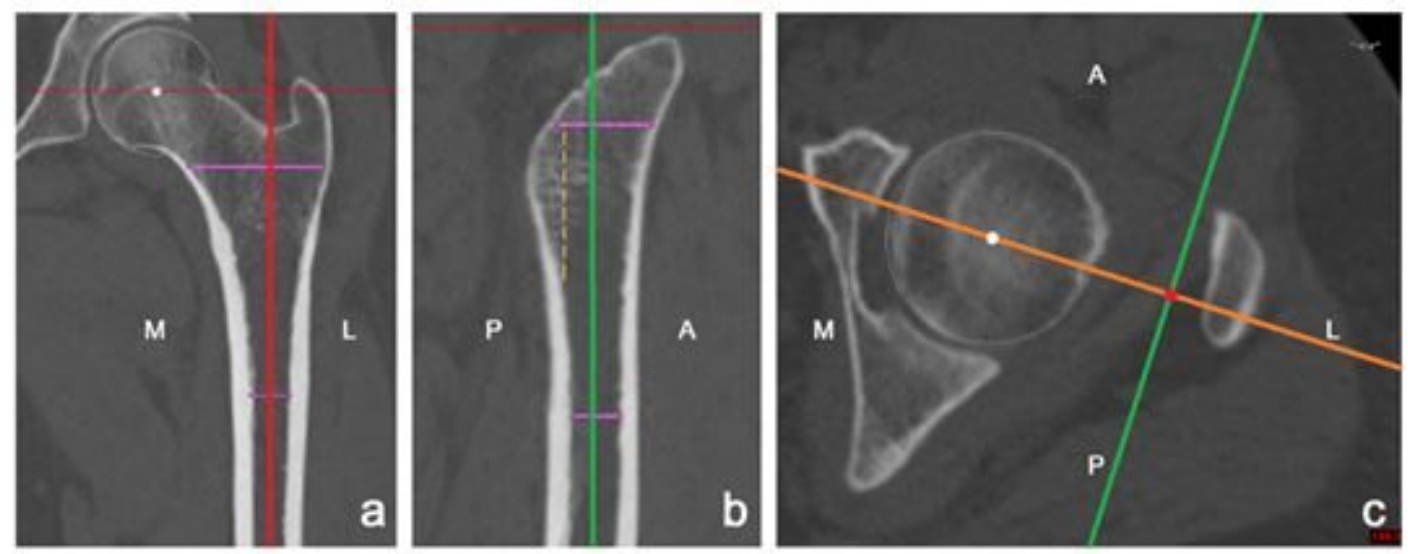

\section{Figure 1}

a) Frontal plane: plane through the fitted spherical center of the femoral head and the axis of the medullary cavity. White dot: spherical center of the femoral head; solid pink lines: coronal medial diameter at $20 \mathrm{~mm}$ distal to the lesser trochanteric line (MT-20) and at $60 \mathrm{~mm}$ proximal to the lesser trochanteric line (MT+60); green line: projection of the sagittal plane; red line: projection of the frontal plane. b) Sagittal plane: plane through the central axis of the medullary cavity and perpendicular to the coronal/horizontal plane. Pink solid lines: MT-20 and MT+60; orange line: frontal plane projection; red line: frontal plane projection; pink dashed line: demarcation between dense cancellous bone and the medullary cavity behind the medullary cavity. c) Horizontal plane: plane perpendicular to the central axis of the medullary cavity. White dot: spherical center of the femoral head; red dot: projection point of the central axis of the medullary cavity in the frontal plane; green line: projection of the sagittal plane; orange line: projection of the frontal plane. 


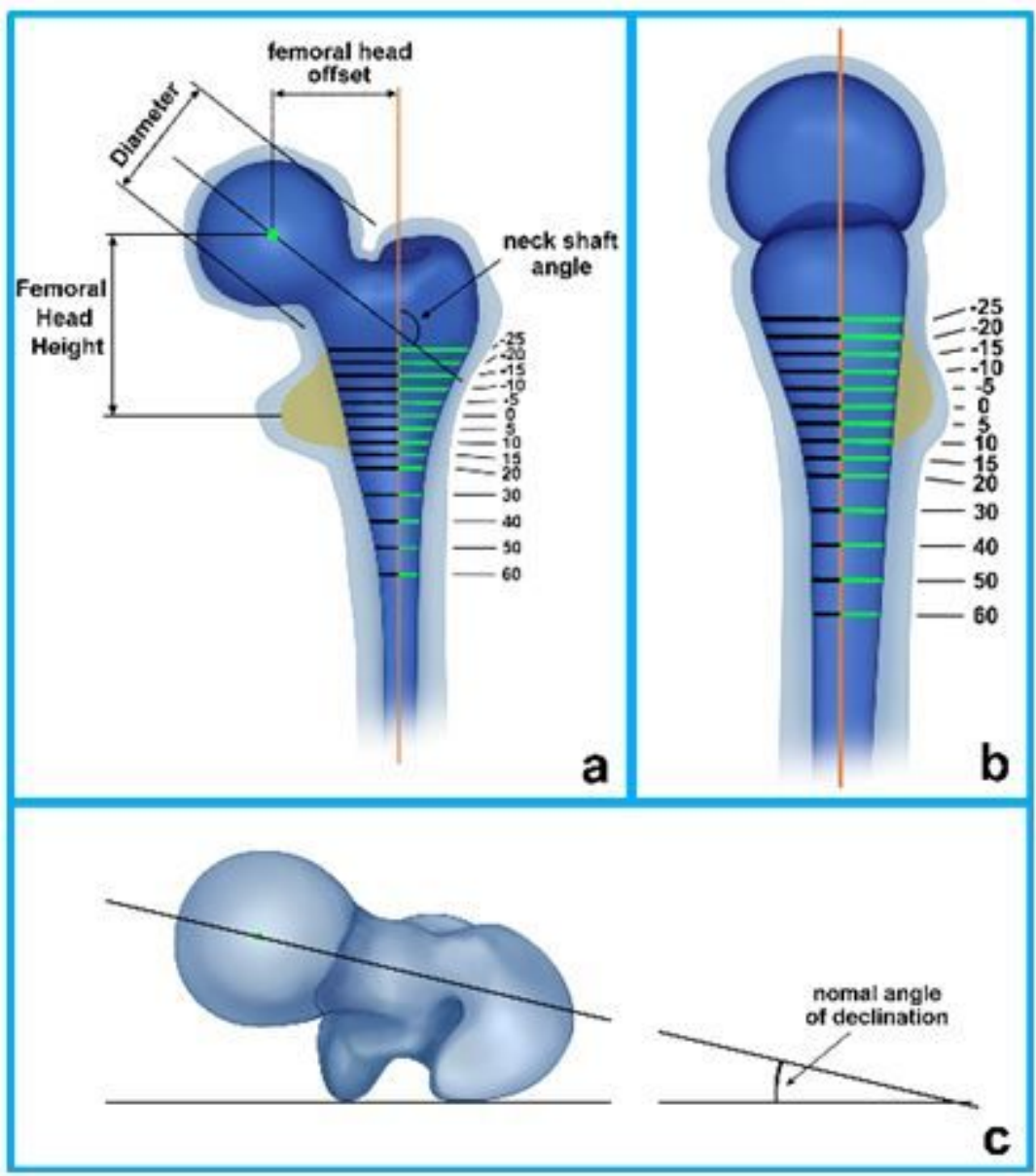

Figure 2

a) Parameters in the frontal plane of the femoral medullary cavity: femoral head diameter, offset, and neck-shaft angle. b) Measured parameters of the sagittal plane of the femoral medullary cavity. c) Measurement of the femoral anteversion angle. The yellow region is the cancellous area with high bone density in the inner and posterior endosteal margin of the metaphysis.

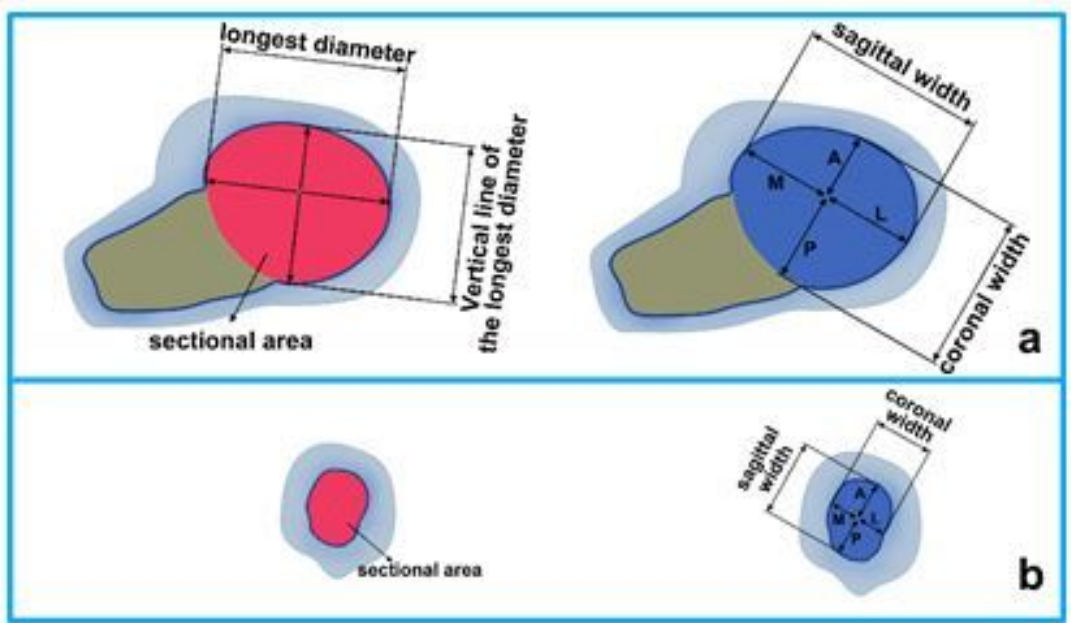

Figure 3 
a) Sectional area of the metaphysis of the medullary cavity showing the coronal medial diameter (M), coronal lateral diameter $(\mathrm{L})$, sagittal anterior diameter $(A)$, and sagittal posterior diameter $(P)$. b) Sectional area of the diaphysis of the medullary cavity showing $M, L, A$, and $P$. The yellow region is the cancellous area with high bone density in the inner and posterior endosteal margin of the metaphysis.

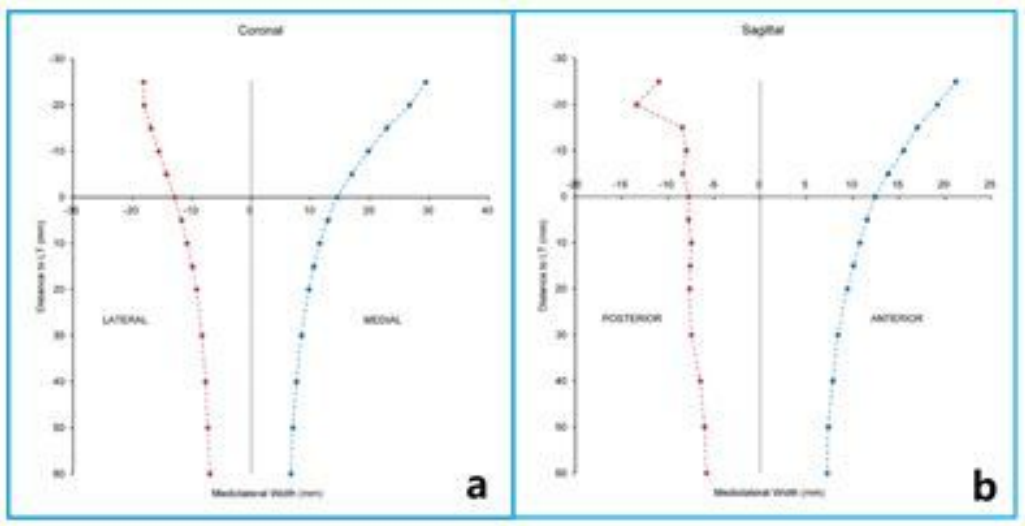

\section{Figure 4}

a) Coronal proximal medullary cavity morphology. The medial and lateral medullary cavity gradually narrowed from proximal to distal, and the trend of narrowing gradually decreased. The medial diameter was wider than the lateral diameter in the metaphysis of the medullary cavity. b) Sagittal proximal medullary cavity morphology. The sagittal anterior diameter of the medullary cavity gradually narrowed from proximal to distal, but the sagittal posterior diameter at T-25 (25 $\mathrm{mm}$ proximal to the lesser trochanteric line) was smaller than that at T-20 (20 mm proximal to the lesser trochanteric line), and the sagittal posterior diameter tended to remain constant from T-10 $(10 \mathrm{~mm}$ proximal to the lesser trochanteric line) to $\mathrm{T}+30$ (30 $\mathrm{mm}$ distal to the lesser trochanteric line).

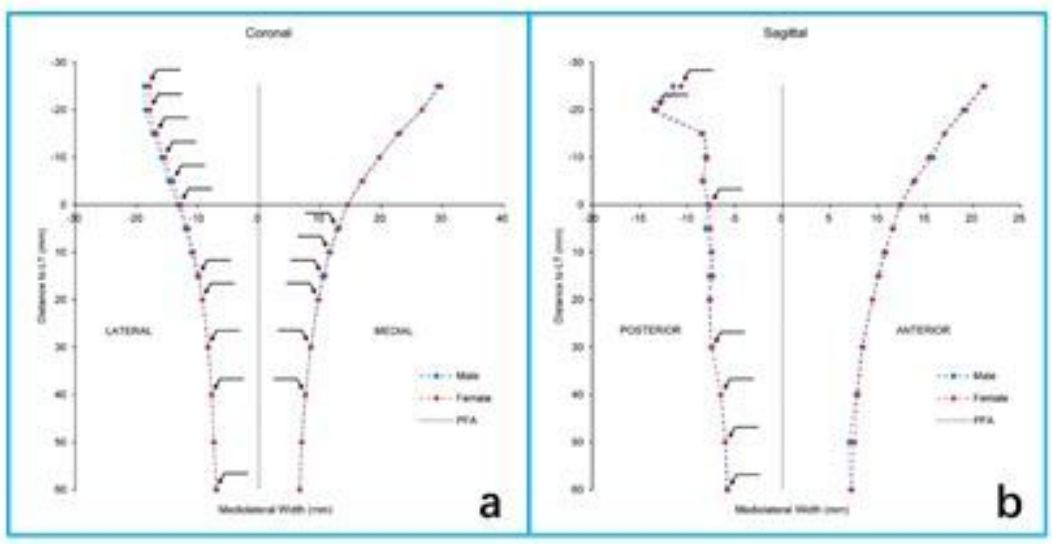

\section{Figure 5}

The medullary cavity was wider in men than women. There were no significant differences between men and women in a) the coronal medial diameter of the metaphysis, and b) the sagittal anterior diameter. At the other levels, the parameters significantly differed between the sexes. 